\title{
Coronabedingte Änderungen in der Agenda der SGAIM
}

Verschiebung SGAIM-Frühjahrskongress 2020 wegen der aktuellen Situation rund um das Coronavirus

Der Vorstand der Schweizerischen Gesellschaft für Allgemeine Innere Medizin (SGAIM) hat nach reiflicher Überlegung und in Abstimmung mit dem wissenschaftlichen Komitee entschieden, den 5. Frühjahrskongress vom 27.-29. Mai 2020 im Congress Center Basel auf das kommende Jahr zu verschieben.

Moderatorenkurs

Ein neuer Moderatorenkurs QZ findet am 26./27. Februar 2021 statt; der Kurs vom Mai 2020 wurde abgesagt.

Delegiertenversammlung

Die Delegiertenversammlung der SGAIM vom 30. April 2020 findet im Rahmen eines Zirkularbeschlusses statt.

Generalversammlung

Die Generalversammlung der SGAIM wird im Rahmen des Herbstkongresses stattfinden, weitere Informationen folgen.
Preisverleihung SGAIM-Preis/SGAIM Foundation

Die Gewinner/-innen werden im Mai schriftlich informiert und auch die entsprechenden Preisgelder ausbezahlt. Die eigentliche Preisverleihung und die Würdigung der Arbeiten werden im festlichen Rahmen des Herbstkongresses stattfinden.

Herbstkongress 17.-18. September 2020, Lugano

Unter dem Motto "Medizin in Bewegung" richtet sich der Kongress sowohl an Spitalinternist/-innen als auch an Hausärztinnen und -ärzte und bietet eine Vielzahl an Fortbildungsmöglichkeiten in der Medizin, die sich stetig weiterentwickelt und uns in die Zukunft begleitet. Am besten, Sie reservieren sich das Datum schon heute.

Bitte beachten Sie die Informationen zu den Veranstaltungen auf www.sgaim.ch 